\title{
Proposta de Rápida Recuperação por Redução de Log
}

\author{
Luiz Gustavo C. Xavier ${ }^{1}$, Fernando Luís Dotti ${ }^{2}$, \\ Cristina Meinhardt ${ }^{1}$, Odorico M. Mendizabal ${ }^{1}$ \\ ${ }^{1}$ Instituto de Informática e Estatística \\ Universidade Federal de Santa Catarina (UFSC) \\ ${ }^{2}$ Faculdade de Informática \\ Pontíficia Universidade Católica do Rio Grande do Sul (PUCRS) \\ l.gustavo.x@posgrad.ufsc.br, fernando.dotti@pucrs.br, \\ \{odorico.mendizabal, cristina.meinhardt\}@ufsc.br
}

\begin{abstract}
Resumo. Logs são cruciais no desenvolvimento de aplicações distribuídas tolerantes a falhas. Este trabalho apresenta uma abordagem para acelerar a recuperação em protocolos de recuperação baseados em log. A abordagem explora a eliminação consciente de comandos de maneira pouco intrusiva à aplicação, resultando em arquivos de logs menores sem ferir a consistência de estado da aplicação, permitindo uma rápida recuperação.
\end{abstract}

\section{Introdução}

Mecanismos de log desempenham um papel central no desenvolvimento de sistemas de gerenciamento de banco de dados [Mohan et al. 1992], replicação e protocolos de coordenação e integração de dados [Kreps et al. 2011]. Porém, além de adicionar custos durante a operação normal, o processamento de um $\log$ de comandos afeta diretamente o tempo de recuperação. Mecanismos de log tradicionais (SL) normalmente não se beneficiam da semântica de comandos da aplicação, sendo desconhecido para o protocolo de recuperação se um resultado de comando é substituído posteriormente ou se sua execução não modifica o estado da aplicação em recuperação.

Neste trabalho, é apresentada uma abordagem para acelerar a recuperação em protocolos baseados em $\log$. Na abordagem de $\log$ proposta (PL) os comandos são analisados em lotes, e a redução é realizada considerando somente os comandos contidos no lote. Uma vez que PL se baseia na semântica da aplicação para o processo de redução, é assumido o modelo de dados de um armazenamento de valor-chave executando comandos de leitura e escrita de uma única variável. Nesse caso, as operações de leitura e escritas subsequentes são exemplos de comandos desnecessários, onde apenas o último comando de escrita de uma variável por lote precisa ser mantido para atingir um estado consistente.

\section{Avaliação Experimental e Considerações Finais}

Para avaliar a rapidez obtida com a execução do protocolo de recuperação apresentado, são analisados os arquivos de log gerados em diferentes configurações de intervalo em relação à abordagem tradicional, comparando o número de comandos e o tamanho total dos arquivos. Os resultados apresentados foram obtidos com a execução de um protótipo de aplicação de armazenamento chave-valor desenvolvida em Go, com a geração de carga de trabalho usando o Yahoo! Cloud Serving Benchmark (YCSB). 
Na Figura 1a é ilustrada a redução obtida no número de comandos para cada carga de trabalho, onde o tamanho do lote é configurado em 1, 10, 100 e 1000 comandos. Para todas as cargas de trabalho, a abordagem SL registrou todos os $10^{6}$ comandos no log. $\mathrm{O}$ mesmo pode ser dito para o cenário de $100 \%$ escritas YCSB-AW, apresentando o mesmo número de comandos que SL independente do tamanho do lote. Isso é explicado pela carga de trabalho de somente escritas com chaves de acesso distribuídos uniformemente. Em um cenário onde há grande incidência de escritas subsequentes sobre uma mesma variável (YCSB-AWL), é possível perceber que quanto maior o tamanho do lote, maior a chance de tais operações serem identificadas e descartadas durante os procedimentos de $\log$. Nesta mesma carga de trabalho, observa-se um ganho de $32 \%$ menos de comandos no PL1000. Todos os comandos são eliminados para YCSB-C, uma carga de 100\% leituras, e obtém-se mais de $90 \%$ de redução em YCSB-B e YCSB-D.

A Figura $1 b$ mostra as reduções de tamanho total do log em comparação com SL. Nos cenários PL1, a abordagem proposta apresenta uma penalidade de $\approx 17 \%$ no tamanho total do log para cargas de trabalho YCSB-AW e YCSB-AWL e um aumento de $5 \%$ para YCSB-A. Isso é explicado pelo fato de que o PL gera um novo arquivo de $\log$ com seus metadados em cada redução de lote, e a soma de todos esses arquivos gerados possui um tamanho maior quando comparado a um único arquivo gerado em SL. Esse efeito não é significativo para outras cargas de trabalho, em que reduções de tamanho são mostradas para cargas de trabalho com leitura intensiva. Para tamanhos de lote $\geq 10$ comandos, YCSB-A mantém uma redução de $\approx 20 \%$. YCSB-AWL mostra benefícios incrementais em lotes maiores, com uma redução de $30 \%$ nos logs para PL1000. Diferenças maiores podem ser vistas para YCSB-C, B e D, onde uma diminuição de quase $100 \%$ é mostrada para o primeiro e $\approx 80 \%$ para os demais em tamanhos de lote de 1000 comandos.

Este resumo apresentou uma abordagem de $\log$ que explora a semântica da aplicação para eliminar comandos desnecessários, provendo indícios de uma recuperação mais rápida. Trabalhos futuros irão avaliar o impacto real desta técnica na recuperação.

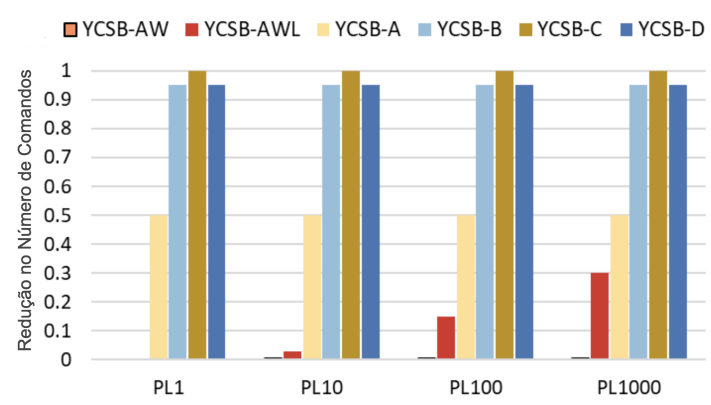

(a) Redução no número de comandos

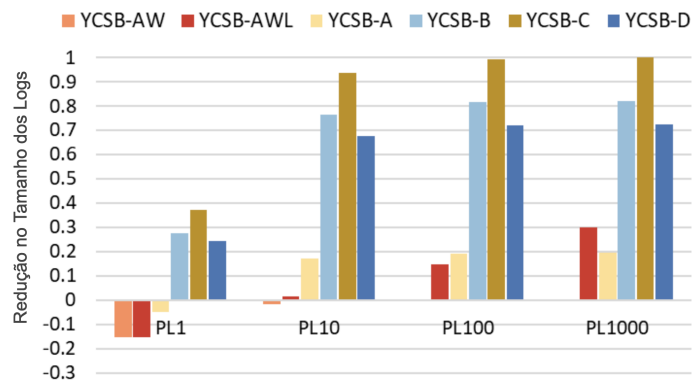

(b) Redução no tamanho dos $\log s$

Figura 1. Avaliação dos arquivos de log gerados. Dados normalizados para SL.

\section{Referências}

Kreps, J., Narkhede, N., Rao, J., et al. (2011). Kafka: A distributed messaging system for log processing. In Proceedings of the NetDB, volume 11, pages 1-7.

Mohan, C., Haderle, D., Lindsay, B., Pirahesh, H., and Schwarz, P. (1992). Aries: a transaction recovery method supporting fine-granularity locking and partial rollbacks using write-ahead logging. ACM Transactions on Database Systems (TODS), 17(1):94-162. 